\title{
MLK3 regulates bone development downstream of the faciogenital dysplasia protein FGD1 in mice
}

\author{
Weiguo Zou, ${ }^{1}$ Matthew B. Greenblatt, ${ }^{1}$ Jae-Hyuck Shim,,1 Shashi Kant, ${ }^{2}$ Bo Zhai, ${ }^{3}$ Sutada Lotinun, ${ }^{4}$ \\ Nicholas Brady, ${ }^{1}$ Dorothy Zhang Hu, ${ }^{1}$ Steven P. Gygi, ${ }^{3}$ Roland Baron, ${ }^{4}$ Roger J. Davis, ${ }^{2}$ \\ Dallas Jones, ${ }^{1}$ and Laurie H. Glimcher ${ }^{1}$

\begin{abstract}
'Department of Immunology and Infectious Diseases, Harvard School of Public Health, Department of Medicine, Harvard Medical School, and Ragon Institute of MGH, Harvard and MIT, Boston, Massachusetts, USA. ${ }^{2}$ Howard Hughes Medical Institute and Program in Molecular Medicine, Department of Biochemistry and Molecular Biology, University of Massachusetts Medical School, Worcester, Massachusetts, USA ${ }^{3}$ Department of Cell Biology, Harvard Medical School, Boston, Massachusetts, USA. ${ }^{4}$ Department of Oral Medicine Infection and Immunity,
\end{abstract} \\ Harvard Dental School, Boston, Massachusetts, USA.
}

\begin{abstract}
Mutations in human FYVE, RhoGEF, and PH domain-containing 1 (FGD1) cause faciogenital dysplasia (FGDY; also known as Aarskog syndrome), an X-linked disorder that affects multiple skeletal structures. FGD1 encodes a guanine nucleotide exchange factor (GEF) that specifically activates the Rho GTPase CDC42. However, the mechanisms by which mutations in FGD1 affect skeletal development are unknown. Here, we describe what we believe to be a novel signaling pathway in osteoblasts initiated by FGD1 that involves the MAP3K mixed-lineage kinase 3 (MLK3). We observed that MLK3 functions downstream of FGD1 to regulate ERK and p38 MAPK, which in turn phosphorylate and activate the master regulator of osteoblast differentiation, Runx2. Mutations in FGD1 found in individuals with FGDY ablated its ability to activate MLK3. Consistent with our description of this pathway and the phenotype of patients with FGD1 mutations, mice with a targeted deletion of $M l k 3$ displayed multiple skeletal defects, including dental abnormalities, deficient calvarial mineralization, and reduced bone mass. Furthermore, mice with knockin of a mutant Mlk3 allele that is resistant to activation by FGD1/CDC42 displayed similar skeletal defects, demonstrating that activation of MLK3 specifically by FGD1/CDC42 is important for skeletal mineralization. Thus, our results provide a putative biochemical mechanism for the skeletal defects in human FGDY and suggest that modulating MAPK signaling may benefit these patients.
\end{abstract}

\section{Introduction}

In 1970, Aarskog described an X-linked recessive syndrome characterized by an upturned nose, short stature, multiple dental defects, delayed skeletal age, and multiple bone malformations (1, 2). Later work confirmed these observations, naming the disorder faciogenital dysplasia (FGDY) or Aarskog-Scott syndrome and identified the gene mutated as FYVE, RhoGEF, and PH domain-containing 1 (FGD1) (3). FGD1 encodes a member of the guanine nucleotide exchange factor (GEF) family, which catalyses the exchange of GDP for GTP and promotes the activity of Rho family GTPases (3-6). More than 16 distinct FGD1 mutations have been reported to cosegregate with FGDY. These mutations include deletions and premature truncations, implying that a loss of FGD1 function underlies FGDY $(6,7)$. Despite these insights into the genetic basis of FGDY, it remains unclear how FGD1 affects bone development.

Expression analysis demonstrates that FGD1 is highly expressed in osteoblasts, suggesting that FGD1 signaling plays a critical role in osteoblast differentiation and function (8). Microinjection studies show that FGD1 specifically activates CDC42, a member of the Rho (Ras homology) family of GTPase proteins $(4,5)$. A

Authorship note: Weiguo Zou and Matthew B. Greenblatt contributed equally to this work.

Conflict of interest: Laurie H. Glimcher declares that she is a member of the board of directors of and holds equity in Bristol-Myers Squibb.

Citation for this article: J Clin Invest. 2011;121(11):4383-4392. doi:10.1172/JCI59041. recent study demonstrates that overexpression of FGD1/CDC42 regulates the differentiation of human mesenchymal cells into osteoblasts in vitro (9). Although impaired FGD1/CDC42 signaling is suspected to be responsible for the skeletal defects in faciogenital dysplasia, the downstream effectors of FGD1/CDC42 and the mechanism by which mutations in FGD1 might affect skeletal mineralization remain unknown.

Because FGD1 is a CDC42 GEF, understanding signaling downstream of CDC42 is key to defining the overall role of FGD1 in osteoblasts. Numerous signaling pathways have been reported to be activated downstream of CDC42, including the p21-activated kinases, the activated CDC42 kinases, a PAR3/6/PKC $\zeta$ complex involved in cell polarity, the myotonic dystrophy kinase-related CDC42-binding kinases, Wiskott-Aldrich syndrome protein, and the mixed-lineage kinases (MLKs) (10-19). Among them, MLK3 belongs to a family of mitogen-activated protein kinase kinases (MAP3K). MLK3 has been best studied as an upstream activator of JNK MAPK. Knockdown of Mlk3 in vitro suggests that MLK3 may have additional roles upstream of p38 or ERK, though the physiologic contexts in which this occurs are unclear $(20,21)$. Recently, we demonstrated that MAPK signaling is essential for bone formation and that TAK1, another MAP3K, can regulate osteoblast function through a TGF- $\beta$-activated kinase 1 -MAPK kinase 3/6-p38-runtrelated transcription factor 2 (TAK1-MKK3/6-p38-Runx2) axis (22). Interestingly, absence of dorsal midline hair has been reported in $M l k 3^{-/-}$mice (23), and absence of midline hair on the vertex has 
A

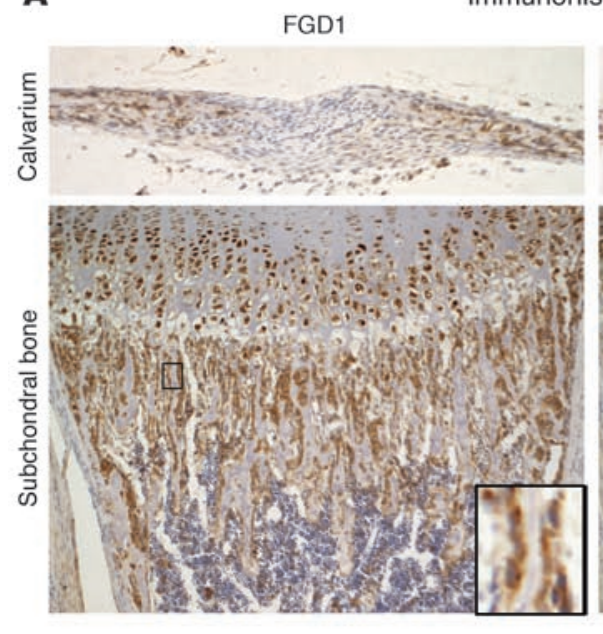

C

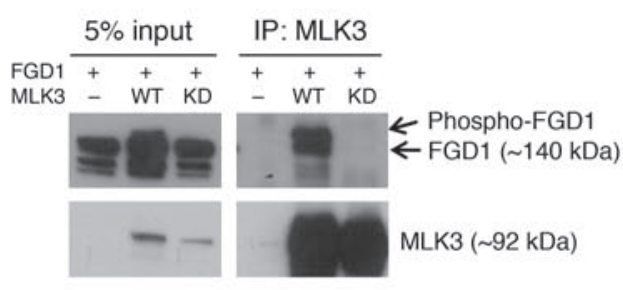

MLK3

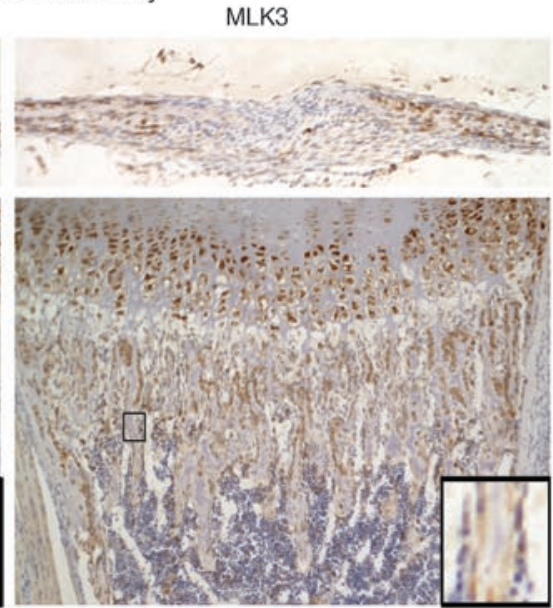

B MLK3 ++++

CDC42 - + - +

FGD1 - - + +

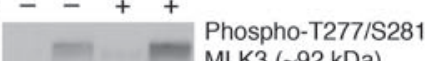

MLK3 ( 92 kDa)

Phospho-T277/S28

MLK3 ( 92 kDa)

-

-aforat Nonspecific ( 80 kDa)

$=\mathrm{FGD} 1(\sim 140 \mathrm{kDa})$
Phospho-T277/S281

MLK3 $(\sim 92 \mathrm{kDa})$

MLK3 ( 92 kDa)

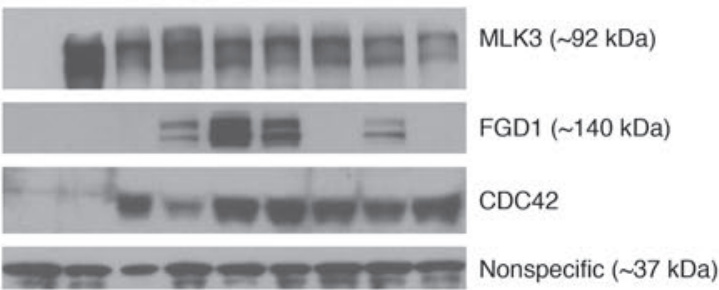

\section{Figure 1}

FGD1 and CDC42 are upstream activators of MLK3. (A) FGD1 and MLK3 expression were examined by immunostaining of adjacent serial sections of proximal tibia and calvaria from 5-day-old mice. Original magnification, $\times 100$. (B) Activation of MLK3 was assessed by blotting for phospho-T277/S281 MLK3 after transfection of 293T cells with constructs encoding proteins as indicated. (C) Coimmunoprecipitation experiments were conducted in 293T cells transfected with FGD1 and MLK3 expression constructs. MLK3 was immunoprecipitated from cell lysates with anti-MLK3 antibody, followed by Western blot analysis with anti-FGD1 antibody. KD, kinase-dead. (D) Activation of MLK3 was assessed as in B following transfection of different FGD1 mutants.

also been reported in patients with FGDY (24). These observations suggest that further investigation into the link between FGD1 and MLK3 in skeletal mineralization would likely yield new insights into both FGDY and signaling pathways in osteoblasts.

\section{Results}

FGD1 and CDC42 are upstream activators of MLK3. To explore the hypothesis that MLK3 is a key mediator of the effects of FGD1 on the skeletal system and, by extension, the human FGDY phenotype, we examined the expression of FGD1 and MLK3 in skeletal tissues. Immunohistochemical staining of serial sections showed robust coexpression of FGD1 and MLK3 in osteoblastlining cells in subchondral trabecular bone, in adjacent osteocytes, and in osteoblasts along the osteogenic fronts in which active growth takes place in the calvarium (Figure 1A). We next sought to determine whether FGD1 can activate MLK3 in vitro. Coexpression of FGD1 and MLK3 in $293 \mathrm{~T}$ cells induced activation of MLK3, as measured by phosphorylation of the critical activa- tion loop sites $\operatorname{Thr}^{277}$ and $\operatorname{Ser}^{281}$ (Figure 1B and ref. 25). FGD1 can also synergize with CDC42 to further increase CDC42-mediated MLK3 activation, indicating that FGD1 and CDC42 collaborate to induce MLK3 activation (Figure 1B). The ability of FGD1 to activate MLK3 suggests that MLK3 and FGD1 might physically interact. Immunoprecipitation of MLK3, followed by Western blotting for FGD1, revealed that these 2 proteins interact when both are ectopically expressed in $293 \mathrm{~T}$ cells (Figure 1C). Interestingly, we found that, compared with that of WT MLK3, the MLK3 kinase-dead mutant displayed a much weaker interaction with FGD1. Coexpression of WT MLK3 with FGD1 induced a mobility shift in FGD1, and this lower mobility band was enriched after immunoprecipitation of MLK3. These data suggest that MLK3 can induce FGD1 phosphorylation and that this phosphorylation event enhances the interaction between MLK3 and FGD1. To examine whether disruption of MLK3 activation might contribute to FGDY, the ability of 5 FGD1 mutants isolated from patients with FGDY (R443H, R522H, 528-insC, R610Q, 1362-insG) to 
A

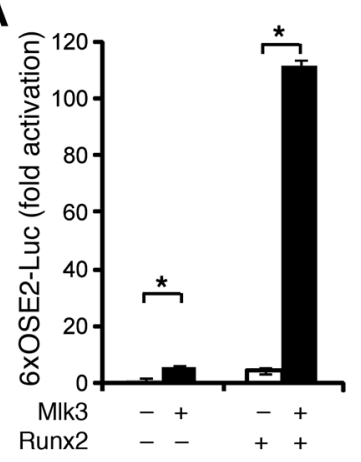

B

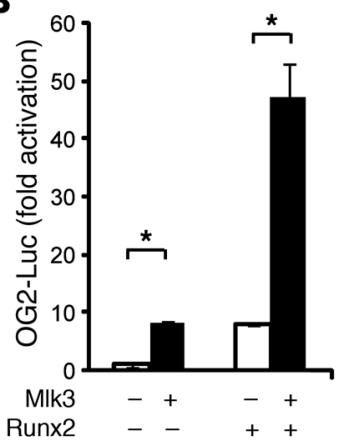

C

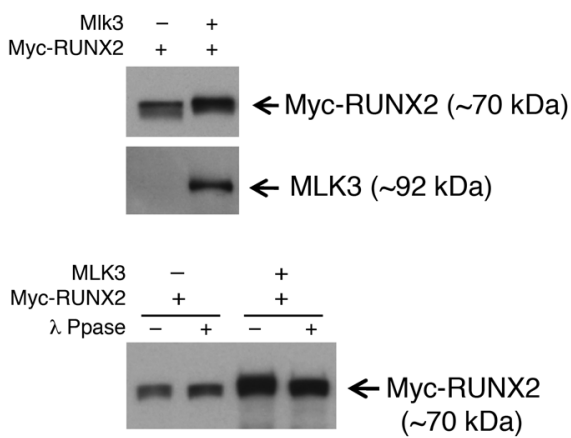

D

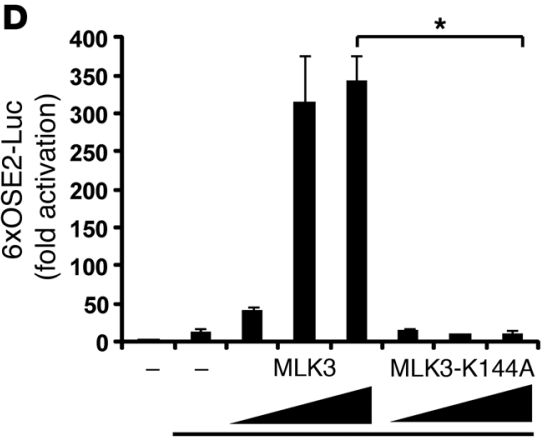

RUNX2

$\mathbf{F}$

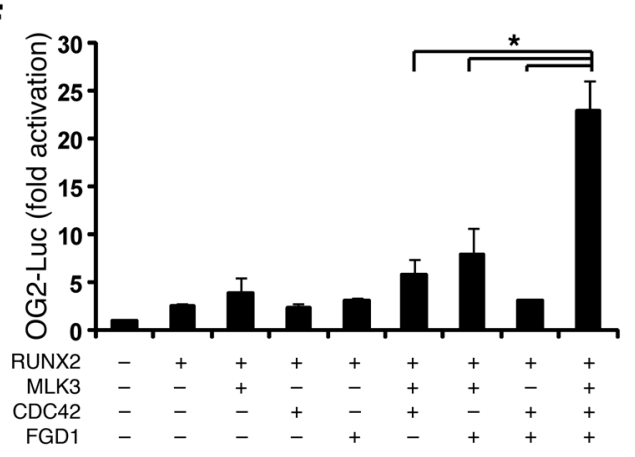

E

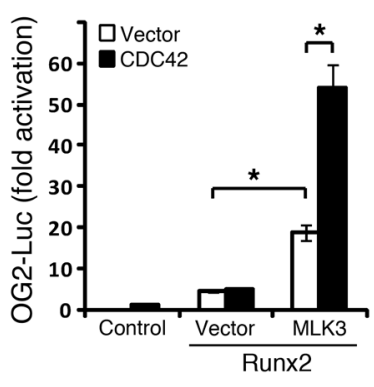

G

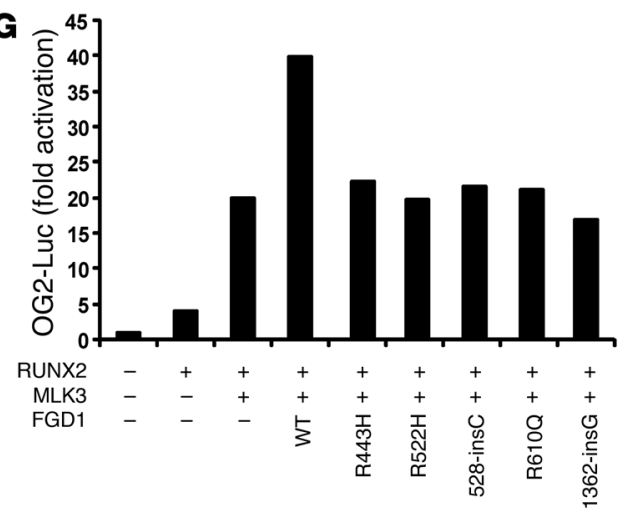

Figure 2

FGD1, CDC42, and MLK3 collaborate to drive Runx2 phosphorylation and activation. (A and B) MLK3 expression increases Runx2 activity, as measured by analyzing Runx2-responsive (A) OSE2-luc activity and (B) OG2-luc activity $\left.{ }^{\star} P<0.01\right)$. (C) MLK3-induced Runx2 mobility shift was assessed by SDS-PAGE and Western blotting after transfection of 293T cells with MLK3 and Myc-Runx2 (top). MLK3-induced Runx2 mobility shift was reversed by $\lambda$ phosphatase ( $\lambda$ Ppase) (bottom). (D) MLK3, but not the kinase-inactive mutant MLK3-K144A, increases Runx2-responsive OSE2-luc activity in a dose-dependent manner ( $\left.{ }^{*} P<0.01\right)$. (E) CDC42 can collaborate with MLK3 to increase Runx2-responsive OG2-luc activity $\left({ }^{\star} P<0.01\right)$. (F) FGD1, CDC42, and MLK3 can synergize to increase Runx2-responsive OG2-luc activity $\left({ }^{*} P<0.01\right)$. (G) WT FGD1, but not FGD1 mutants, can increase Runx2-responsive OG2-luc activity.

activate MLK3 was examined $(7,26)$. Expression of these FGD1 proteins bearing any of the FGDY patient mutations reduced or ablated the robust activation of MLK3 by WT FGD1 (Figure 1D).

FGD1, CDC42, and MLK3 collaborate to drive Runx2 phosphorylation and activation. Previously, we demonstrated that the p38 MAPK pathway is critical in osteoblast differentiation and bone development through its regulation of Runx 2 activity (22). We next examined whether FGD1, CDC42, and MLK3 can also regulate Runx2 activity and osteoblast differentiation. Coexpression of MLK3 with Runx2 resulted in dramatic activation of the Runx2-responsive luciferase reporter constructs OSE2-luc (Figure 2A) and OG2- luc (Figure 2B). Coexpression of MLK3 together with Myc-Runx2 resulted in a shift in the electrophoretic mobility of Runx2, which was reversible by treatment with $\lambda$ phosphatase (Figure $2 \mathrm{C}$ ), confirming that MLK3 can indeed induce Runx2 phosphorylation. A kinase-inactive MLK3 mutant, MLK3-K144A, was unable to increase Runx2 activity, demonstrating that MLK3 kinase activity is required to support Runx2 activation (Figure 2D). Consistent with the observation that FGD1 and CDC42 are competent to induce MLK3 activation (Figure 1B), coexpression of CDC42 with MLK3 increased Runx2 activity over that of MLK3 alone (Figure 2E), and FGD1 and CDC42 synergized with MLK3 to further 
increase Runx2 activity (Figure 2F). In addition, the ability of WT FGD1 to potentiate MLK3-induced Runx2 activity was reduced or ablated by the FGD1 mutations found in FGDY (Figure 2G). Thus, MLK3 activation downstream of FGD1/CDC42 results in Runx2 phosphorylation and activation, and this pathway is disrupted by the mutations found in human FGDY.

Runx2 activity is intimately linked to osteoblast differentiation and function (27-29). Given that MLK3 regulates Runx2 activity, we examined whether MLK3 can promote osteoblast functions. FGD1, CDC42, and MLK3 are all expressed in human pluripotent mesenchymal stem cells (hMSCs) (Supplemental Figure 1A; supplemental material available online with this article; doi:10.1172/ JCI59041DS1), which can be differentiated into osteoblasts under proper culture conditions $(29,30)$. Consistent with MLK3 increasing Runx2 transcriptional activity, ectopic expression of MLK3, but not $\mathrm{MLK}^{\mathrm{K} 144 \mathrm{~A}}$, in hMSCs promoted osteoblast differentiation, determined by both Fast Blue staining for alkaline phosphatase (ALP) and Von Kossa staining for mineralization (Supplemental Figure 2, A and B). Likewise, knockdown of MLK3 blocked osteoblast differentiation. This effect could be replicated with 2 independent shRNAs and correlated with the level of reduction in MLK3 protein (Supplemental Figure 2, C-E). Moreover, expression of mouse MLK3, which is not targeted by the shRNAi constructs directed against human MLK3, was able to rescue the effects of MLK3 knockdown, in terms of both ALP activity (Figure 3A) and mineralization capacity (Supplemental Figure $2 \mathrm{~F}$ ), proving the specificity of the knockdown results.

$M L K 3$ promotes osteoblast differentiation through $p 38$ and ERK MAPK. To probe the mechanisms by which MLK3 influences osteoblast differentiation, MLK3-expressing cells were treated with inhibitors of different MAPKs, including p38, ERK, or JNK MAPK pathways. Only cotreatment with both ERK and p38 inhibitors could reverse the ability of MLK3 to promote osteoblast differentiation, in terms of both ALP activity (Figure 3, B and C) and mineralization capacity (Figure 3C), indicating that both $\mathrm{p} 38$ and ERK pathways are important for MLK3-mediated osteoblast differentiation. Both ERK and p38 MAPK pathways have been implicated in the phosphorylation and activation of Runx $2(22,31)$. Consistent with a role for MLK3 upstream of both ERK and p38, mass spectroscopy analysis reflected that coexpression of MLK3 with Runx2 in 293T cells induced Runx2 phosphorylation at both previously identified ERK-mediated (S28, S282, S301, and S319) and p38-mediated (S28, S244, S301, S319, and S472) phosphorylation sites (Figure 3D, Supplemental Figure $3 \mathrm{~A}$, and Supplemental Table 1). Additionally, we believe S395 was identified as a novel Runx2 phosphorylation site induced by MLK3, although it is unclear whether its phosphorylation is mediated by p38 or ERK or whether this reflects the activity of a novel pathway downstream of MLK3. We mutated 3 of the overlapping phosphorylation sites (S28, S301, and S309) to alanine and found that MLK3induced Runx2 activity is dramatically impaired (Figure 3E). Thus, MLK3 functions as an upstream activator of p38 and ERK MAPKs in osteoblasts, and p38/ERK, in turn, phosphorylates and activates Runx2 to promote osteoblast differentiation.

Mlk3 $3^{-/}$osteoblasts showed a reduction in the phosphorylation of both p38 and ERK1/2 by both Western blotting ex vivo (Figure 4A) and immunohistochemistry for phospho-p38 and phosphoERK in vivo (Supplemental Figure 4A). Previously, we demonstrated a role for TAK1 in mediating p38 activation downstream of BMP2/7 in osteoblasts (22). Mlk3 $3^{-/}$osteoblasts showed reduced levels of both p38 and ERK activation after BMP2/7 stimulation (Supplemental Figure 4B). These results confirm that MLK3 is a physiologic activator of ERK and p38 MAPK in osteoblasts in vivo, validating previous observations that MLK3 functions upstream of ERK and p38 in vitro (21). The expression of MKK3 and MKK6, 2 p38 signaling intermediates, was unchanged in the absence of MLK3 (Supplemental Figure 4A), indicating that reduced p38 phosphorylation in the absence of MLK3 is not due to altered MAP2K expression. Previously, we showed that p38-mediated Runx 2 phosphorylation increased the ability of Runx 2 to interact with CREB-binding protein (CBP) (22). To assess the effects of MLK3 on the interaction between Runx 2 and CBP, Myc-Runx 2 was expressed in WT and $M l k 3^{-/}$calvarial osteoblasts. Consistent with a function for MLK3 in activating p38, which, in turn, promotes the CBP/Runx2 interaction, immunoprecipitation with anti-CBP and blotting for Myc-Runx2 revealed reduced levels of CBP/Runx2 interaction in the absence of MLK3 (Supplemental Figure 4C).

Thus, MLK3 is critical for osteoblast differentiation and mineralization in vitro, and this activity is mediated by the ability of MLK3 to regulate Runx2 both via ERK and p38 MAPK-induced phosphorylation.

Mlk3 $3^{--}$mice display impaired skeletal mineralization and spontaneous tooth fracture. To extend these in vitro observations, we next examined the contribution of MLK3 to skeletal development in vivo by analyzing the bone phenotype of $\mathrm{Mlk}^{-/-}$mice (Figure 4, B-I). Many patients with FGDY display a generalized delay in the ossification of multiple bony structures. Compared with that of WT controls, Mlk $3^{-/-}$mice displayed delayed calvarial development, with postnatal day 5 pups showing hypomineralization of the frontal bone (Figure $4 \mathrm{~B})$, and $100 \%$ of 3 - to 4 -week-old mice showing persistent defects along the coronal suture (Figure 4B). To quantitate the extent of the hypertelorism, the distance between the left and right insertion sites of the zygomatic arch was measured and found to be increased in Mlk $3^{-/-}$mice, a finding consistent with the hypertelorism characteristic of FGDY (Supplemental Figure 5A and ref. 32). Additionally, we found that 3.5-week-old Mlk3-/- mice were osteopenic, with an approximately $50 \%$ reduction in femoral trabecular bone volume/ total volume (BV/TV) analyzed, which was largely accounted for by a decrease in trabecular number (Figure 4, C and D). Consistent with this decrease in skeletal mineralization, expression of characteristic osteoblast marker genes, osteocalcin (Ocn, also known as $O C)$ and collagen I $\alpha$ (Col1a1), was reduced in osteoblasts in vivo (Figure 4E). This decrease was specific, as levels of the marker gene secreted phosphoprotein 1 (also known as osteopontin [Opn]) were unchanged (Figure 4E). Confirming that the osteopenia observed in $M l k 3^{-/-}$mice reflects impaired osteoblast activity, histomorphometric analysis also demonstrated reduced bone mass and decreased osteoblast synthetic capacity. Compared with that of WT controls, Mlk3 ${ }^{-/}$mice at 3.5 weeks of age had significant reductions in both bone volume and bone formation rate (Figure 4, F-H, and Supplemental Table 2). Mlk3 $^{-/-}$mice had a $49 \%$ decrease in cancellous bone volume and trabecular number, with a concomitant increase in trabecular spacing. Bone formation rate, when expressed per bone surface, bone volume, or tissue volume, was decreased (by 28\%,29\%, and $61 \%$, respectively). The number of osteoblasts per bone surface area and the percentage of actively mineralizing bone surface were not significantly changed, arguing that the decrease in the bone formation rate and mineral apposition rate (reduced $21 \%$ ) reflects an intrinsic defect in osteoblast matrix synthetic capacity as opposed to a generalized paucity of osteoblasts.

In addition to skeletal defects, defects in tooth development are also present in patients with FGDY $(2,33,34)$. In support of a role 
A
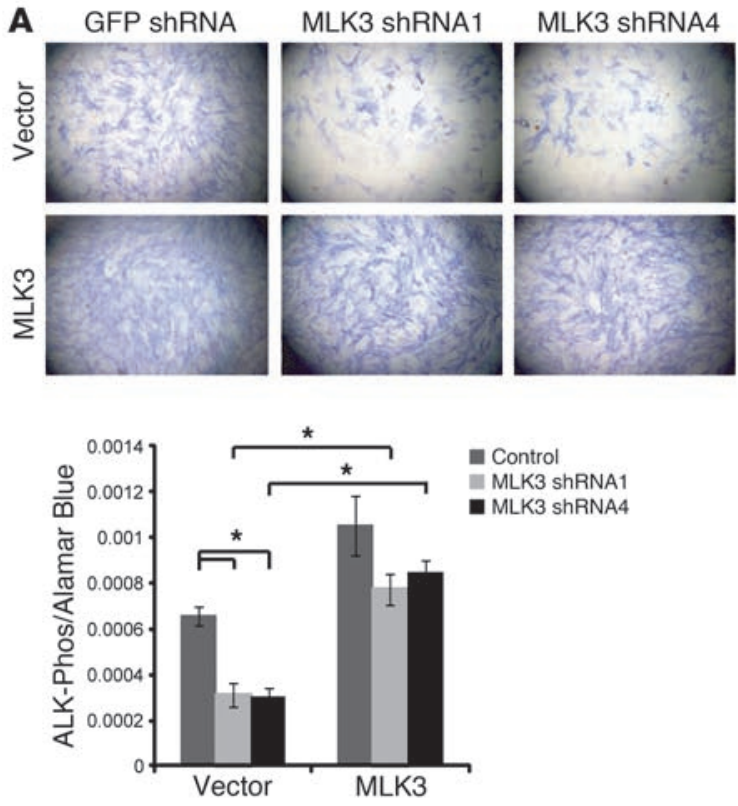

Control
MLK3 shRNA1
MLK3 shRNA4

MLK3 shRNA4

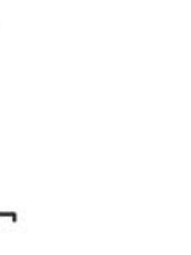

B

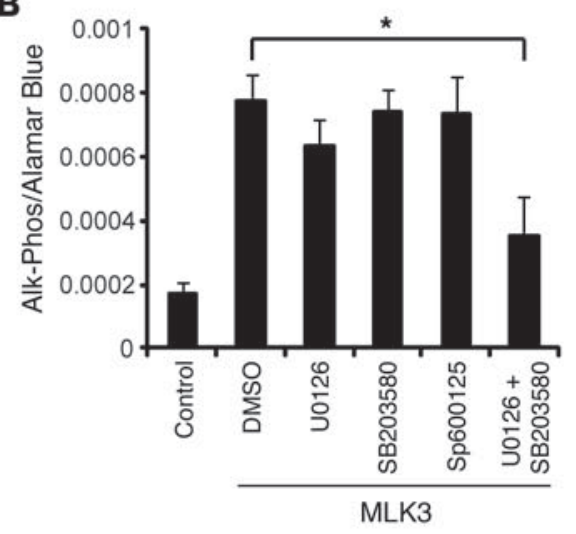

c

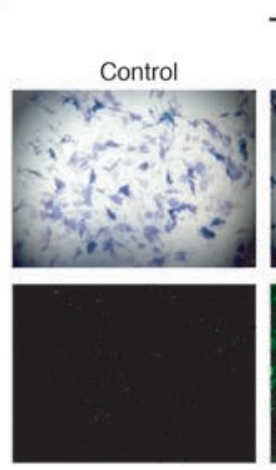


A

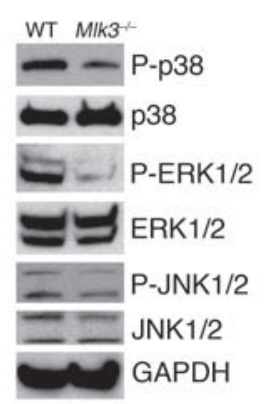

D

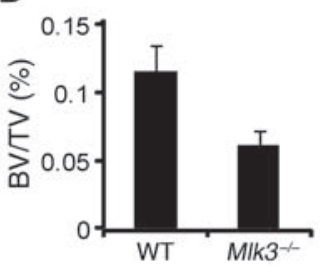

E WT
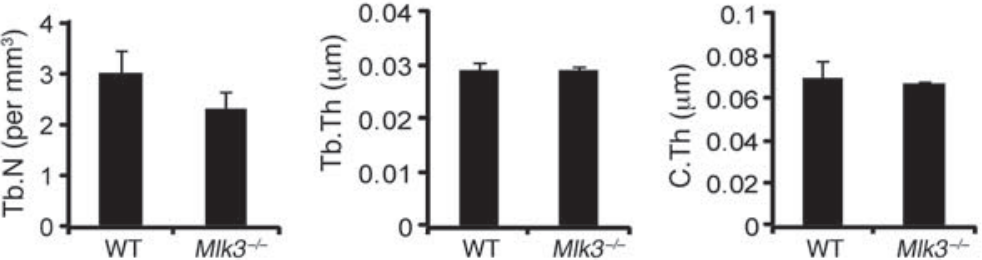

Mlk3--

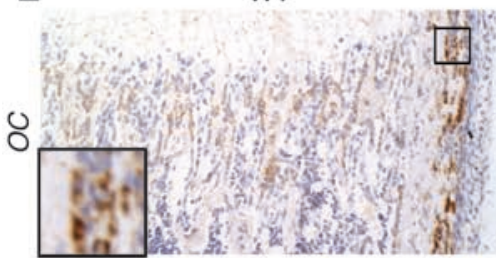

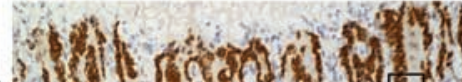

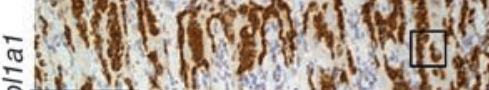
o $\cos ^{4} \mathrm{f}^{3}$
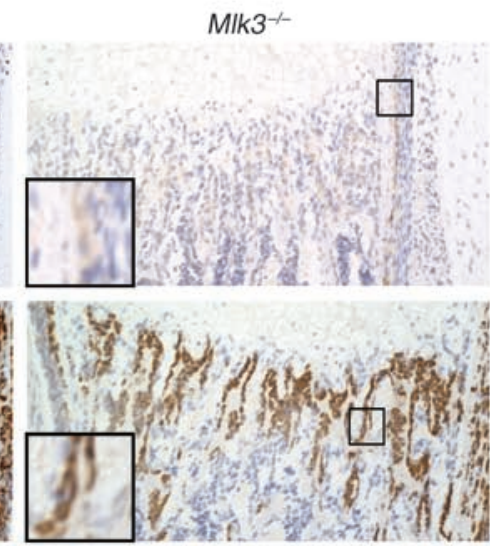

$\mathbf{F}$
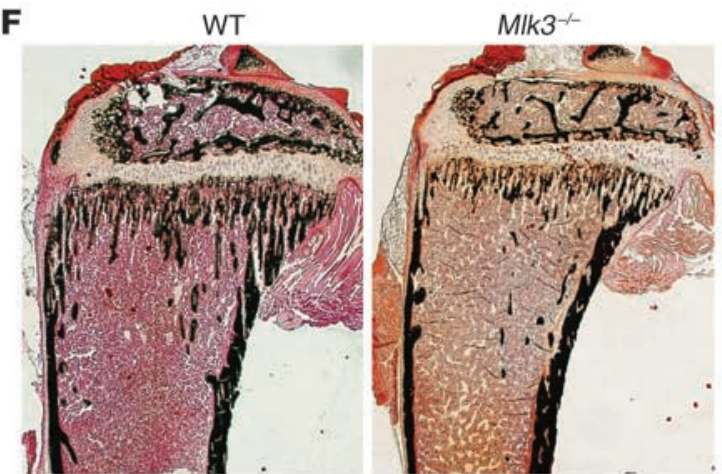
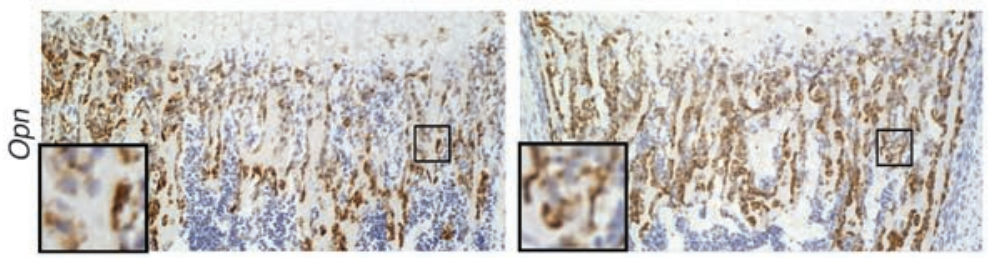

G

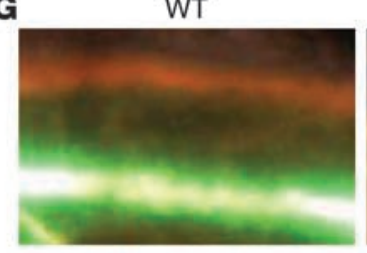

MIk3--

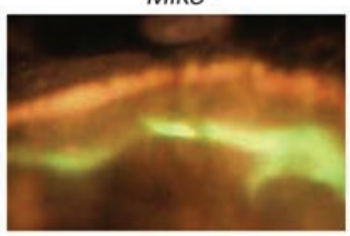

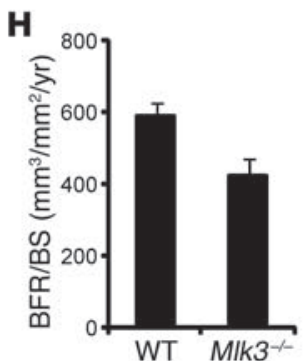
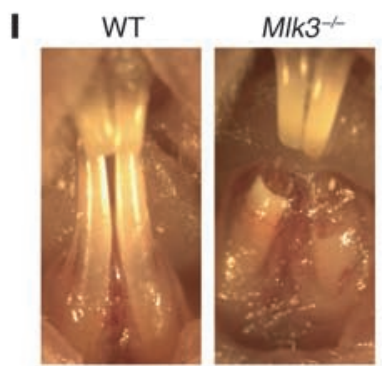


\section{Figure 4}

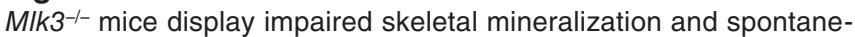
ous tooth fracture. (A) Phosphorylation of p38, JNK, and ERK was evaluated by Western blot. Cell lysates were prepared from calvarial osteoblasts isolated from $\mathrm{Mlk3}^{-1-}$ and control mice and were cultured for 6 days in vitro under osteoblast differentiation conditions. (B) Representative 3D reconstructions of calvarial bone from 5-day-old and 3-week-old $\mathrm{Mlk3}^{-1-}$ mice and control WT mice. (C and D) $\mu \mathrm{CT}$ analysis of distal femurs from 3-week-old female $\mathrm{Mlk3}^{-1-}$ mice and control mice. (C) Representative 3D reconstructions of distal femur trabecular bone and midshaft cortical bone. (D) Quantitative parameters were BV/TV $(P<0.005)$, trabecular number (Tb.N; $P<0.005)$, trabecular thickness (Tb.Th; $P=\mathrm{NS}$ ), and cortical thickness (C.Th; $P=\mathrm{NS}$ ). (E) In situ hybridization for the indicated probes on proximal tibia of 5-dayold $\mathrm{Mlk3}^{-/-}$and control mice. High-magnification insets are provided. Original magnification, $\times 100$. (F) Von Kossa staining of proximal tibia from 3.5-week-old $\mathrm{Mlk3}^{--}$and control mice. Histomorphometric analysis showed a decrease in BV/TV of $3.60 \% \pm 0.67 \%$ for WT mice and $1.78 \% \pm 0.28 \%$ for $\mathrm{Mlk3}^{-/-}$mice $(P<0.05)$ and a decrease in trabecular number per mm of $1.48 \pm 0.25$ for WT mice and $0.75 \pm 0.11$ for Mlk3-mice $(P<0.05)$. Original magnification, $\times 40$. $(\mathbf{G})$ Representative fluorescent micrography pictures of Calcein/demeclocycline-labeled mineralization fronts in proximal tibiae bone from 3.5-week-old mice. Original magnification, $\times 400$. (H) Quantification of bone formation rate (BFR, bone formation rate; BS, bone surface area), measured with calcein and demeclocycline double labeling $(P=0.021)$. (I) Representative pictures of 3 - to 4 -week-old $\mathrm{Mlk3}^{-1-}$ and control mice, showing spontaneous fracture of the mandibular incisors.

for FGD1 in tooth development, immunohistochemistry demonstrated that FGD1 was expressed in both ameloblasts and odontoblasts, which produce tooth enamel and dentin (Supplemental Figure 5B). Interestingly, 5-day-old $M l k 3^{-/-}$mice displayed a marked reduction in the volume of tooth dentin (Supplemental Figure 5, $\mathrm{C}-\mathrm{E})$, and all $\mathrm{Mlk3^{-/- }}$ mice developed spontaneous fracture of the incisors by 3 to 4 weeks of age (Figure 4I). Histologic characterization of the teeth of $\mathrm{Mlk3}^{-/-}$mice revealed a disorganization of the basolateral surface of the odontoblast layer (Supplemental Figure 5F). To better understand the role of MLK3 in odontoblast cells, we infected the T4-4 odontoblast cell line with Mlk3-specific shRNA lentivirus and found that depletion of MLK3 in odontoblast cells reduced their mineralization capacity and expression of the odontoblast marker gene dentin sialophosphoprotein (Dspp) and dentin matrix component dentin matrix acidic phosphoprotein 1 (Dmp1) (Supplemental Figure 6, A and B, and ref. 35). Consistently, expression of DSPP was reduced in MLK3-knockout mice in vivo (Supplemental Figure 6C). Thus, in vivo and in vitro analyses support the conclusion that MLK3 is a physiologic regulator of osteoblast differentiation and bone development. Additionally, MLK3 plays a critical role in the development of tooth dentin, and absence of MLK3 results in severe dental defects.

Knockin mice expressing a mutant MLK3 resistant to FGD1 activation demonstrate the physiologic relevance of the FGD1/CDC42/MLK3 pathway. A motif-based search analysis revealed that MLK3 can potentially interact with CDC42 via a CDC42/Rac interactive binding (CRIB) region $(36,37)$. According to this, mutation of the CRIB motif (I492A, S493A) might render MLK3 refractory to activation by FGD1. We found that the MLK3 CRIB mutant (hereafter referred to as the $M L K 3^{m 3 c b}$ mutant) was resistant to FGD1-induced MLK3 activation, as measured by MLK3 phosphorylation (Figure $5 \mathrm{~A})$. When compared with WT MLK3, MLK3 $3^{m 3 c b}$ did not fully support Runx 2 activation, as measured by Runx2-induced luciferase activity (Figure 5B). We next asked whether MLK3 is a physiologically relevant downstream effector of FGD1 in vivo by examining mice homozygous for a knockin allele of the CRIB mutant MLK3. These mice, hereafter referred to as $M l k 3^{m 3 c b}$ mice, displayed a skeletal phenotype similar to that of $M l k 3^{-/-}$mice, demonstrating that activation of MLK3 by FGD1/CDC42 is essential for MLK3 function in vivo. As seen in $M l k 3^{-1-}$ mice, all $M l k 3^{m 3 c b}$ mice examined showed impaired calvarial ossification, most prominently in the frontal bone at 3 to 4 weeks of age (Figure 5C). Likewise, Mlk3 $3^{m 3 c b}$ mice showed a similar degree of reduction in trabecular BV/TV (Figure 5, D and E) as that of $M l k 3^{-/-}$mice. In situ hybridization also revealed a similar spectrum of impairments in osteoblast-specific gene expression, with a modest reduction in Col1a1, Bsp, and $O C$ and relative sparing of Opn expression (Figure 5F). All Mlk3 $3^{m 3 c b}$ mice examined also showed spontaneous fracture of the incisors (Figure 5G). Thus, $M l k 3^{m 3 c b}$ mice recapitulate the defects seen in Mlk3 ${ }^{-/-}$mice with striking fidelity, supporting the idea that FGD1/ CDC42 is the predominant upstream activator of MLK3 in vivo.

Lastly, to directly demonstrate the importance of the FGD1/ CDC42/MLK3 signaling axis in osteoblast differentiation, we differentiated WT and Mlk3 ${ }^{m 3 c b}$ bone marrow stromal cells into osteoblasts. Consistent with the defects observed in vivo, Mlk3 $3^{m 3 c b}$ osteoblasts displayed impaired differentiation, as evidenced by decreased ALP activity and mineralization (Figure 5H). Additionally, the expression of several osteoblast marker genes, such as Col1a1, Bsp, and OC, was reduced (Supplemental Figure 7A). Atf4 also showed a modest but significant reduction in expression. Thus, $M l k 3^{m 3 c b}$ mice display substantial defects in osteoblast functions, both in vivo and in vitro, validating the importance of the FGD1/CDC42/MLK3 pathway.

\section{Discussion}

The rarity of patients with FGDY, combined with the difficulty of obtaining human skeletal tissue, has hampered progress in understanding the molecular mechanisms underlying FGDY. Here, we provide evidence that FGD1 genetic lesions causative for FGDY disrupt a signaling cascade through CDC42 and MLK3, and MLK3 in turn activates Runx2 through p38 and ERK MAPK. Complimenting this biochemical analysis, in vivo genetic ablation of this pathway via either an Mlk3-knockout allele or knockin of an Mlk3 allele that is resistant to FGD1 activation both result in a nearly identical set of skeletal defects. These observations establish a FGD1/CDC42/MLK3/p38/ERK/RUNX2 signaling axis as a physiologic regulator of bone development in vivo, and this pathway is disrupted by FGD1 mutations found in patients with FGDY. These results provide what we believe to be a novel mechanism for the pathogenesis of human FGDY and suggest that modulating MAPK signaling may be of therapeutic benefit for patients with FGDY.

To address the possibility that this signaling pathway might also regulate osteoclast differentiation, osteoclasts were differentiated from bone marrow cells or splenocytes in vitro. Neither absence of MLK3 nor the MLK3 CRIB mutation had any influence on osteoclast differentiation capacity (Supplemental Figure 7 and ref. 38). Furthermore, $M l k 3^{-/-}$mice had similar numbers of osteoclasts in vivo, and $M l k 3^{m 3 c b}$ mice had unaltered levels of the serum biomarker for osteoclast activity, the collagen-derived CTX peptide (Supplemental Figure 7 and ref. 39). When considered alongside the histomorphometry data demonstrating decreased bone formation rates in $M l k 3^{-/-}$mice, it is likely that the decrease in skeletal mineralization observed in $\mathrm{Mlk}^{-/-}$and $M l k^{m 3 c b}$ mice is attributable to a decrease in osteoblast functional capacity as opposed to an increase in osteo- 
A

MLK3

1492A, FGD1 $\frac{\text { MLK3 }}{-+} \frac{\text { S493A }}{-+}$

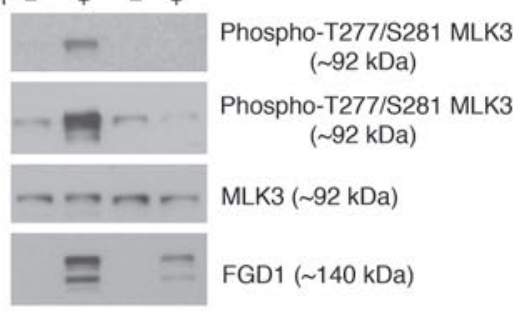

$-\infty$ Nonspecific $(\sim 20 \mathrm{kDa})$
B

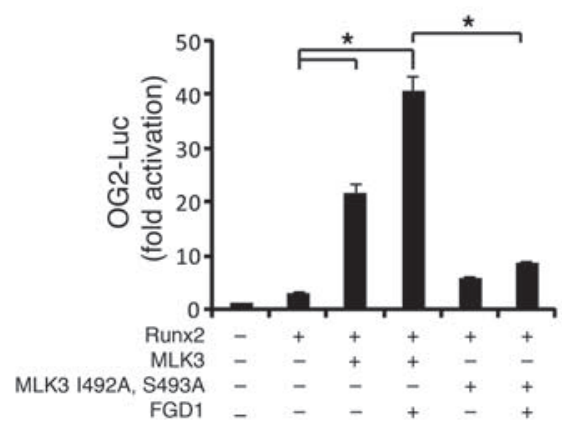

C
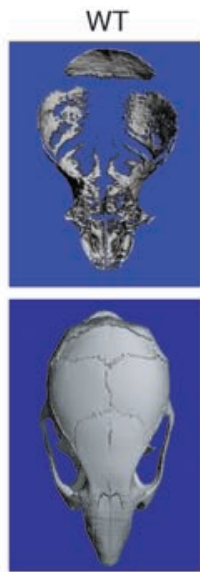

Mlk $3^{m 3 c b}$
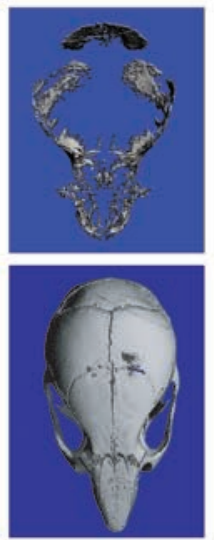

D
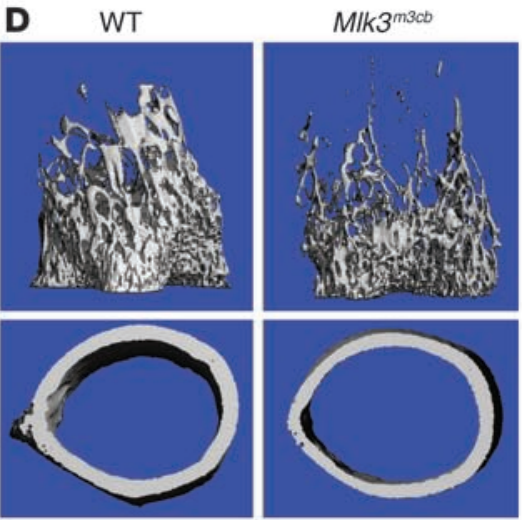
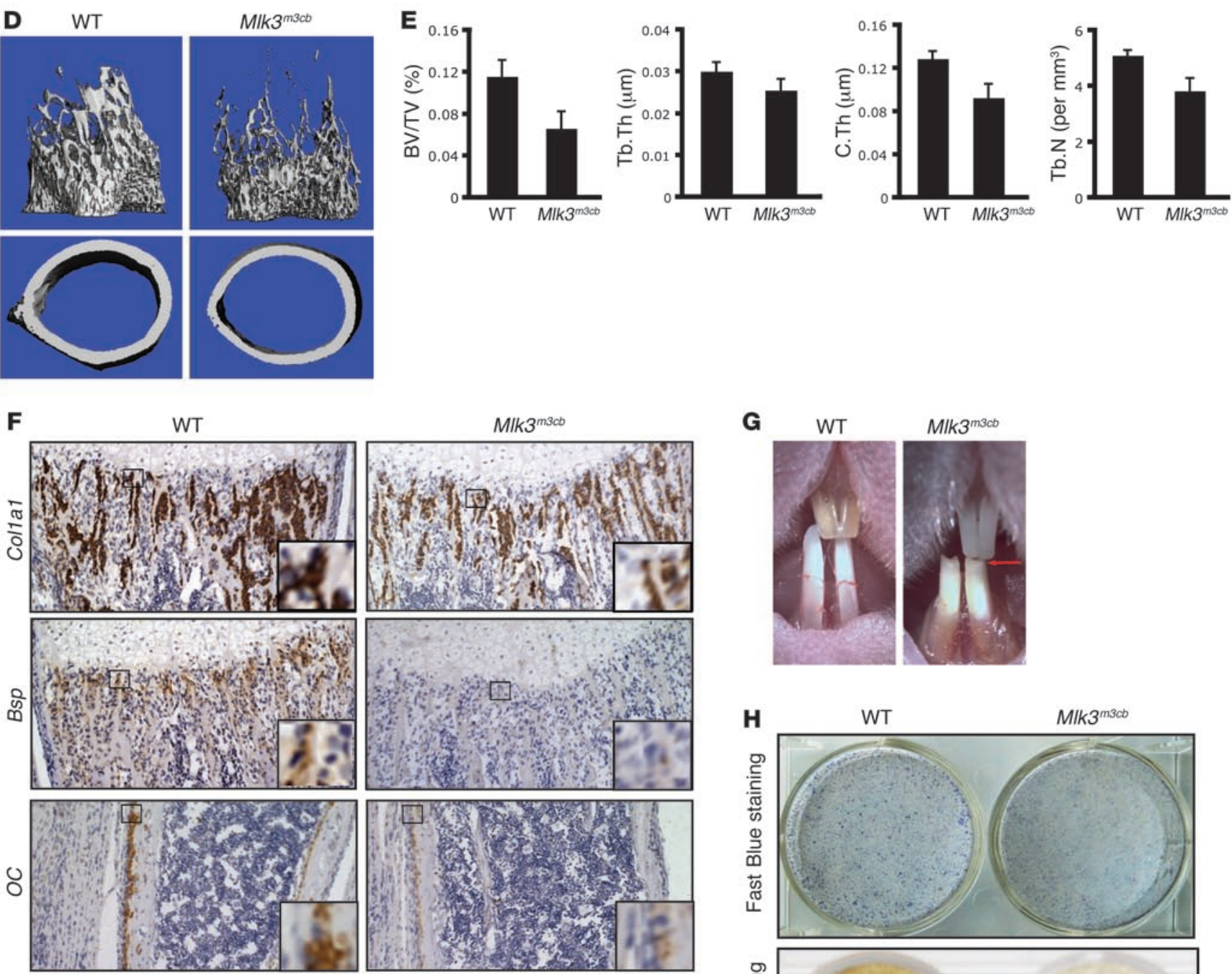

G
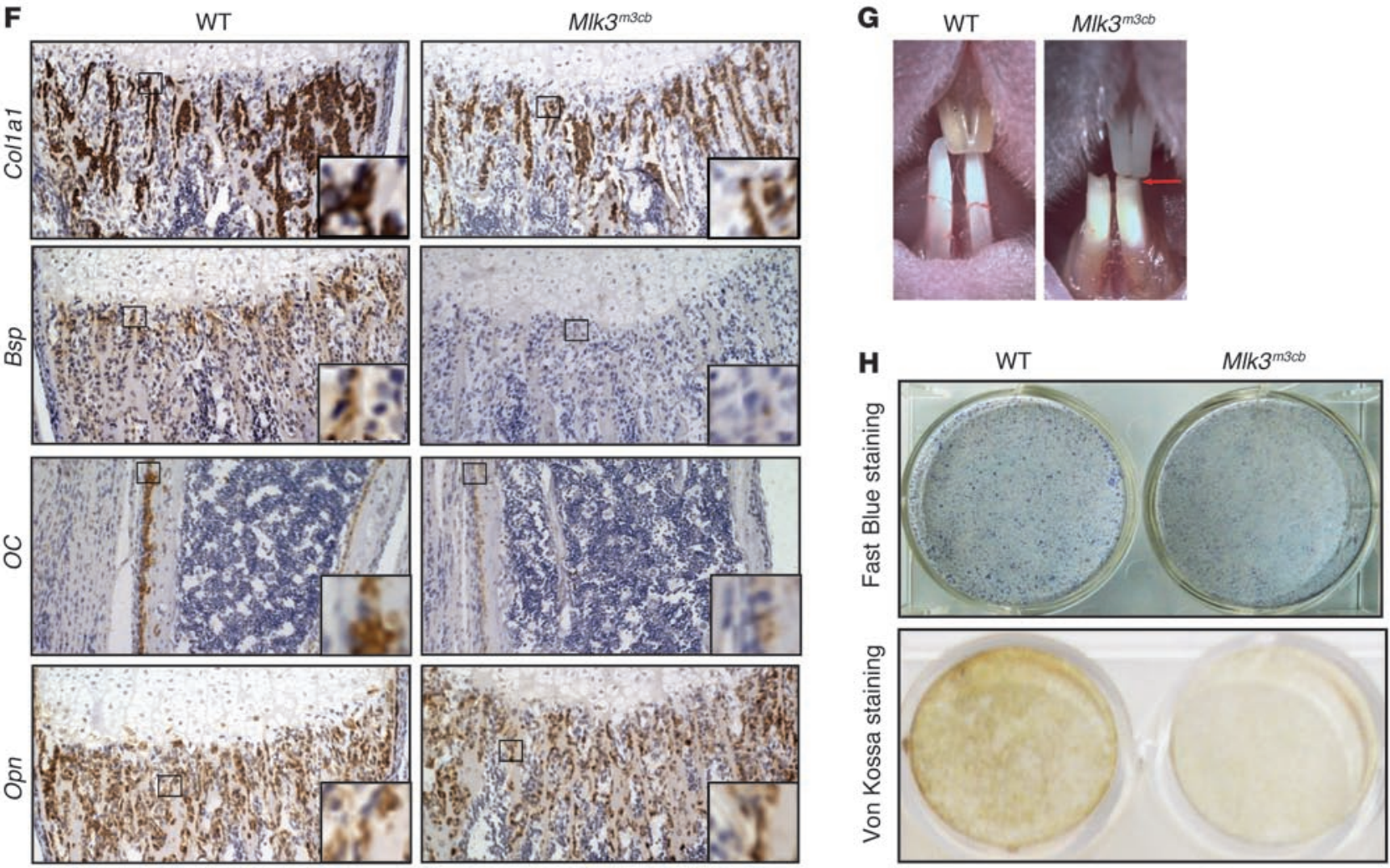


\section{Figure 5}

Mlk3 ${ }^{m 3 c b}$ mice demonstrate the physiologic relevance of the FGD1/ CDC42/MLK3 pathway. (A) FGD1-induced MLK3 T277/S281 phosphorylation was assessed by Western blot after transfection of 293T cells with indicated constructs, including WT MLK3 or MLK3 CRIB mutant (I492A, S493A). The expression of transfected protein was examined by Western blot as well. (B) The effects of FGD1, MLK3, and MLK3 CRIB mutant (I492A, S493A) on Runx2 activity were assessed by analyzing Runx2-responsive OG2-luc activity $\left({ }^{\star} P<0.01\right)$. (C) Representative $3 D$ reconstructions of calvarial bone from 4-day-old and 4-week-old Mlk3m3cb mice and control WT mice. (D and E) $\mu \mathrm{CT}$ analysis of distal femurs from 4-week-old male Mlk3m3cb mice and control mice (BV/TV, $P<0.01$; trabecular thickness, $P<0.05$; cortical thickness, $P<0.01$; trabecular number, $P<0.05$ ). (D) Representative 3D reconstructions of distal femur trabecular bone and midshaft cortical bone. (E) Quantitative parameters were BV/TV, trabecular number, trabecular thickness, and cortical thickness. (F) In situ hybridization for the indicated probes on proximal tibia of 4-day-old MIk3m3cb and control mice. High-magnification insets are provided. Original magnification, $\times 100$. (G) Representative pictures of the incisors of 4-week-old $M I k 3^{m 3 c b}$ and control mice, showing spontaneous fracture of the mandibular incisors. (H) Bone marrow stromal cells were isolated, cultured under differentiation conditions for 6 days, and stained for ALP activity (left). Alternatively, they were cultured for 21 days, and Von Kossa staining was performed for mineralization activity (right).

clast function. Interestingly, CDC42 has recently been implicated in osteoclast differentiation (40). The finding that MLK3 contributes to osteoblast but not osteoclast differentiation downstream of CDC42 suggests substantial tissue-specific differences in the selection of downstream effectors by CDC42. Notably, while the phenotype of the $M l k 3^{m 3 c b}$ mice implicates FGD1 and CDC42 as upstream activators of MLK3 in osteoblasts, it does not exclude that other effectors of CDC42 may also play critical roles in osteoblast differentiation and function. Further study of the methods by which CDC42 effectors are selected in osteoblasts is warranted.

In addition to insights specifically related to FGDY, the examination of MLK3 in bone yields insights relevant to general bone biology. In particular, this study establishes MLK3 alongside TAK1 as a MAP3K relevant to osteoblast differentiation in vivo. Whereas TAK1 only regulates p38 MAPK in osteoblasts, MLK3 contributes to both $\mathrm{p} 38$ and ERK activation, validating previous in vitro studies $(20,21,41)$. Given that both MLK3 and TAK1 contribute to p38 activation, it will be of particular interest to examine how these roles are coordinately regulated and what, if any, overlap exists between the 2 MAP3K in vivo. Of note, whereas both MLK3 and TAK1 were previously well studied as regulators of JNK MAPK, JNK regulation in osteoblasts is independent of either TAK1 or MLK3. Thus, the MAP3K responsible for JNK activation in osteoblasts in vivo remains elusive, and substantial differences exist between MAPK signaling pathways in osteoblasts and those in other cell lineages. This also suggests that defects in JNK signaling are unlikely to be a primary cause of the skeletal manifestations of FGDY.

Intriguingly, studies in Caenorhabditis elegans have identified FGD1 as an evolutionarily conserved mediator of secretory cell development, a finding parallel to our observations in mammalian osteoblasts $(42,43)$. Given that MLKs such as MLK3 are also conserved as far back as C. elegans, this raises the intriguing possibility that an ancient precursor of the FGD1/CDC42/MLK3 pathway regulated the differentiation of secretory cell types in invertebrates and was then co-opted for use in osteoblasts with the advent of vertebrate life. Thus, further examination of MLK3, both in the specific context of human FGDY and in the general context of the evolution of skeletal structures, will be informative.

\section{Methods}

Mice. Mlk $3^{-/-}$mice were previously described (23). Mlk $3^{m 3 c b}$ mice were constructed by knockin of a CRIB motif mutant Mlk3 (I492A, S493A) allele, and construction of this strain is also described in Kant et al. (44). Briefly, a genomic fragment of the Mlk3 gene that includes exons 4-7 was used to introduce 2 point mutations in exon 7 (I492A, S493A) together with a floxed $N e o^{R}$ cassette. Conventional gene targeting was used to produce targeted C57BL/6 ES cells. These ES cells were injected into C57BL/6J blastocysts to produce chimeric mice that were bred to obtain germ-line transmission. The floxed $N e o^{R}$ cassette was excised with Cre recombinase. The heterozygous Mlk3 $3^{m 3 c b}$ animals were crossed to obtain homozygous $M l k 3^{m 3 c b}$ mice. All mice analyzed were on the C57BL/ 6 background. For all control mice analyzed, age- and gender-matched WT C57BL/6 mice housed in the same facility were used.

Plasmids. PCMVsport6-FGD1 and PCMVsport6-MLK3 plasmids were purchased from Openbiosystems. All FGD1 and MLK3 mutations were made by using a QuikChange XL Site-Directed Mutagenesis Kit (Stratagene) according to the manufacturer's instructions. The Runx 2 expression plasmids were the gift of Gerard Karsenty (Columbia University, New York, New York, USA). The multimerized OSE2-luc (6xOSE2) construct and OG2-luc construct were obtained from Bjorn Olsen (Harvard University, Cambridge, Massachusetts, USA).

Osteoblast, osteoclast, and odontoblast differentiation analysis. For ALP staining, osteoblasts were fixed with $10 \%$ neutral buffered formalin and stained with Fast Blue and Naphthol (Sigma-Aldrich). For quantitative determination of ALP activity, osteoblasts were incubated with Alamar Blue and 6.5 $\mathrm{mM} \mathrm{Na}_{2} \mathrm{CO}_{3}, 18.5 \mathrm{mM} \mathrm{NaHCO}_{3}, 2 \mathrm{mM} \mathrm{MgCl}_{2}$, and phosphatase substrate (Sigma-Aldrich). ALP activity was then read with a luminometer (Thermo Electron). For Von Kossa staining of extracellular matrix mineralization, cells were fixed with $10 \%$ neutral formalin buffer and stained with $2.5 \%$ silver nitrate (Sigma-Aldrich). Analysis of osteoclast differentiation and TRAP staining was performed as previously described (45). Serum CTX was measured using the Rat Laps EIA Kit (Immunodiagnostiosystems). The odontoblast T4-4 cell line was cultured under conditions identical to those used for osteoblasts above, except that the medium additionally contained $10 \mathrm{nM}$ dexamethasone. The T4-4 cell line was a gift from Anne George (University of Illinois at Chicago, Chicago, Illinois, USA).

$\mu C T$ analysis. Skulls and femurs were scanned on a Scanco $\mu C T 35$. Femurs and skulls were scanned at 7- and 20-micron resolution, respectively. For analysis of femoral bone mass, a region of trabecular bone 2.1- $\mathrm{mm}$ wide was contoured, starting 280 microns from the proximal end of the distal femoral growth plate. Femoral trabecular bone was thresholded at 211 permille. Femoral cortical bone was thresholded at 350 permille, and calvarium was thresholded at 260 permille. A Gaussian noise filter optimized for murine bone was applied to reduce noise in the thresholded $2 \mathrm{D}$ image. $3 \mathrm{D}$ reconstructions were created by stacking the thresholded $2 \mathrm{D}$ images from the contoured regions.

Luciferase reporter assays. Murine C3H10T1/2 cells grown on 12-well plates were transiently transfected using Effectene (Qiagen) with the Runx2responsive reporter construct (OSE2-luc or OG2-luc) and the Renilla luciferase vector (Promega), together with plasmids encoding MLK3, FGD1, and RUNX2. Total amounts of transfected DNA were kept constant by adding the appropriate control vector. Forty-eight hours after transfection, cells were lysed in 1xPassive Lysis Buffer (Promega), and luciferase activity was measured using the Dual Luciferase Assay Kit (Promega).

RNA extraction and real-time PCR. Total RNA was extracted using TRIzOL (Qiagen), and cDNA was synthesized using the Affinityscript Kit (Agilent) and analyzed by real-time PCR using a Stratagene Mx3005. 
In situ bybridization and immunobistochemistry. In situ hybridization was performed as previously described (22). Briefly, DIG-labeled antisense probes were generated to detect Col1a1, Bsp, OC, Dspp, and Opn mRNA expression. Probes were then hybridized with paraffin sections and visualized using an anti-DIG HRP conjugate system.

Immunohistochemistry was performed as previously described (22). AntiFGD1 antibody was obtained from Aviva Systems Biology; anti-MKK3, phospho-p38, and phospho-ERK antibodies were obtained from Cell Signaling Technology; anti-MKK6 was obtained from Biolegend; and antiMLK3 and anti-Cdc42 were obtained from Santa Cruz Biotechnology Inc.

Statistics. All statistical analysis was performed with the Prism software package. Two-tailed Student's $t$ tests were used throughout. A $P$ value of less than 0.05 was considered significant. All values graphed are mean \pm standard deviation.

Study approval. All mouse experiments were conducted in accordance with a protocol approved by the Institutional Animal Care and Use Committee of the University of Massachusetts Medical School.

\section{Acknowledgments}

The authors thank Gerard Karsenty (Columbia University), Anne George (University of Illinois), Xiuping Wang (Harvard University), and Bjorn Olsen (Harvard University) for providing reagents. This work was supported by NIH grants HD55601 (to L.H. Glimcher), K99AR055668 (to D. Jones), and RO1-CA065861 (to R.J. Davis); by a Yerby Scholar Award from the Harvard School of Public Health (to W. Zou); and by a fellowship from the Arthritis Foundation (to J-.H. Shim). R.J. Davis is an investigator of the Howard Hughes Medical Institute.

Received for publication May 17, 2011, and accepted in revised form August 24, 2011.

Address correspondence to: Laurie H. Glimcher, FXB Rm 205, 651 Huntington Ave., Boston, Massachusetts 02115, USA. Phone: 617.432. 0622; Fax: 617.432.1223; E-mail: lglimche@hsph.harvard.edu.
1. Aarskog D. A familial syndrome of short stature associated with facial dysplasia and genital anomalies. J Pediatr. 1970;77(5):856-861.

2. Halse A, Bjorvatn K, Aarskog D. Dental findings in patients with Aarskog syndrome. Scand J Dent Res. 1979;87(4):253-259.

3. Pasteris NG, et al. Isolation and characterization of the faciogenital dysplasia (Aarskog-Scott syndrome) gene: a putative Rho/Rac guanine nucleotide exchange factor. Cell. 1994;79(4):669-678.

4. Olson MF, Pasteris NG, Gorski JL, Hall A. Faciogenital dysplasia protein (FGD1) and Vav, two related proteins required for normal embryonic development, are upstream regulators of Rho GTPases. Curr Biol. 1996;6(12):1628-1633.

5 . Zheng Y, et al. The faciogenital dysplasia gene product FGD1 functions as a Cdc42Hs-specific guanine-nucleotide exchange factor. J Biol Chem. 1996; 271(52):33169-33172.

6. Rossman KL, Der CJ, Sondek J. GEF means go: turning on RHO GTPases with guanine nucleotide-exchange factors. Nat Rev Mol Cell Biol. 2005; 6(2):167-180.

7. Orrico A, et al. Phenotypic and molecular characterisation of the Aarskog-Scott syndrome: a survey of the clinical variability in light of FGD1 mutation analysis in 46 patients. Eur J Hum Genet. 2004;12(1):16-23.

8. Gorski JL, Estrada L, Hu C, Liu Z. Skeletal-specific expression of Fgd1 during bone formation and skeletal defects in faciogenital dysplasia (FGDY; Aarskog syndrome). Dev Dyn. 2000;218(4):573-586.

9. Gao L, Gorski JL, Chen CS. The Cdc42 guanine nucleotide exchange factor FGD1 regulates osteogenesis in human mesenchymal stem cells. Am J Pathol. 2011;178(3):969-974.

10. Joberty G, Petersen C, Gao L, Macara IG. The cellpolarity protein Par6 links Par3 and atypical protein kinase C to Cdc42. Nat Cell Biol. 2000;2(8):531-539.

11. Lin D, Edwards AS, Fawcett JP, Mbamalu G, Scott JD, Pawson T. A mammalian PAR-3-PAR-6 complex implicated in $\mathrm{Cdc} 42 / \mathrm{Rac} 1$ and $\mathrm{PPKC}$ signalling and cell polarity. Nat Cell Biol. 2000;2(8):540-547.

12. Symons $\mathrm{M}$, et al. Wiskott-Aldrich syndrome protein, a novel effector for the GTPase CDC42Hs, is implicated in actin polymerization. Cell. 1996;84(5):723-734.

13. Leung T, Chen XQ, Tan I, Manser E, Lim L. Myotonic dystrophy kinase-related Cdc42-binding kinase acts as a Cdc42 effector in promoting cytoskeletal reorganization. Mol Cell Biol. 1998;18(1):130-140.

14. Manser E, Leung T, Salihuddin H, Tan L, Lim L. A non-receptor tyrosine kinase that inhibits the GTPase activity of p21cdc42. Nature. 1993; 363(6427):364-367.

15. Arias-Romero LE, Chernoff J. A tale of two Paks. Biol Cell. 2008;100(2):97-108.
16. Manser E, Leung T, Salihuddin H, Zhao ZS, Lim L. A brain serine/threonine protein kinase activated by Cdc42 and Rac1. Nature. 1994;367(6458):40-46.

17. Teramoto $\mathrm{H}$, Coso OA, Miyata $\mathrm{H}$, Igishi T, Miki T, Gutkind JS. Signaling from the small GTP-binding proteins $\mathrm{Rac} 1$ and $\mathrm{Cdc} 42$ to the c-Jun $\mathrm{N}$-terminal kinase/stress-activated protein kinase pathway. A role for mixed lineage kinase 3 /protein-tyrosine kinase 1 , a novel member of the mixed lineage kinase family. J Biol Chem. 1996;271(44):27225-27228.

18. Etienne-Manneville S, Hall A. Cdc42 regulates GSK-3beta and adenomatous polyposis coli to control cell polarity. Nature. 2003;421(6924):753-756.

19. Wilkinson S, Paterson HF, Marshall CJ. Cdc42MRCK and Rho-ROCK signalling cooperate in myosin phosphorylation and cell invasion. Nat Cell Biol. 2005;7(3):255-261.

20. Tibbles LA, et al. MLK-3 activates the SAPK/JNK and p38/RK pathways via SEK1 and MKK3/6. EMBO J. 1996;15(24):7026-7035.

21. Chadee DN, Kyriakis JM. MLK3 is required for mitogen activation of B-Raf, ERK and cell proliferation. Nat Cell Biol. 2004;6(8):770-776.

22. Greenblatt MB, et al. The p38 MAPK pathway is essential for skeletogenesis and bone homeostasis in mice. J Clin Invest. 2010;120(7):2457-2473.

23. Brancho D, Ventura JJ, Jaeschke A, Doran B, Flavell RA, Davis RJ. Role of MLK3 in the regulation of mitogen-activated protein kinase signaling cascades. Mol Cell Biol. 2005;25(9):3670-3681.

24. van den Bergh P, Fryns JP, Wilms G, Piot R, Dralands G, van den Bergh R. Anomalous cerebral venous drainage in Aarskog syndrome. Clin Genet. 1984; 25(3):288-294.

25. Leung IW, Lassam N. The kinase activation loop is the key to mixed lineage kinase- 3 activation via both autophosphorylation and hematopoietic progenitor kinase 1 phosphorylation. J Biol Chem. 2001; 276(3):1961-1967.

26. Schwartz CE, et al. Two novel mutations confirm FGD1 is responsible for the Aarskog syndrome. Eur J Hum Genet. 2000;8(11):869-874.

27. Ducy P, Zhang R, Geoffroy V, Ridall AL, Karsenty G. Osf2/Cbfa1: a transcriptional activator of osteoblast differentiation. Cell. 1997;89(5):747-754.

28. Zaidi M. Skeletal remodeling in health and disease. Nat Med. 2007;13(7):791-801.

29. Nishikawa K, et al. Maf promotes osteoblast differentiation in mice by mediating the age-related switch in mesenchymal cell differentiation. J Clin Invest. 2010;120(10):3455-3465.

30. Bais M, et al. Transcriptional analysis of fracture healing and the induction of embryonic stem cellrelated genes. PLoS One. 2009;4(5):e5393.

31. Ge C, Xiao G, Jiang D, Franceschi RT. Critical role of the extracellular signal-regulated kinase-MAPK pathway in osteoblast differentiation and skeletal development. J Cell Biol. 2007;176(5):709-718.

32. Sepulveda W, Dezerega V, Horvath E, Aracena M. Prenatal sonographic diagnosis of Aarskog syndrome. JUltrasound Med. 1999;18(10):707-710.

33. Reddy P, Kharbanda OP, Kabra M, Duggal R. Dental and craniofacial features of Aarskog syndrome: report of a case and review of literature. J Clin Pediatr Dent. 1999;23(2):155-159.

34. Dayal PK, Chaudhary AR, Desai KI, Joshi HN. Aarskog syndrome. A case report. Oral Surg Oral Med Oral Pathol. 1990;69(3):403-405.

35. Hao J, et al. Odontoblast cells immortalized by telomerase produce mineralized dentin-like tissue both in vitro and in vivo. J Biol Chem. 2002; 277(22):19976-19981.

36. Burbelo PD, Drechsel D, Hall A. A conserved binding motif defines numerous candidate target proteins for both Cdc42 and Rac GTPases. J Biol Chem. 1995;270(49):29071-29074.

37. Du Y, Bock BC, Schachter KA, Chao M, Gallo KA. $\mathrm{Cdc} 42$ induces activation loop phosphorylation and membrane targeting of mixed lineage kinase 3. J Biol Chem. 2005;280(52):42984-42993.

38. Nagase Y, et al. Anti-apoptotic molecule Bcl-2 regulates the differentiation, activation, and survival of both osteoblasts and osteoclasts. J Biol Chem. 2009;284(52):36659-36669.

39. Lee SH, et al. v-ATPase V0 subunit d2-deficient mice exhibit impaired osteoclast fusion and increased bone formation. Nat Med. 2006;12(12):1403-1409.

40. Ito $\mathrm{Y}$, et al. Cdc42 regulates bone modeling and remodeling in mice by modulating RANKL/M-CSF signaling and osteoclast polarization. J Clin Invest. 2010;120(6):1981-1993.

41. Chadee DN, Kyriakis JM. A novel role for mixed lineage kinase 3 (MLK3) in B-Raf activation and cell proliferation. Cell Cycle. 2004;3(10):1227-1229.

42. Gao J, Estrada L, Cho S, Ellis RE, Gorski JL. The Caenorhabditis elegans homolog of FGD1, the human Cdc42 GEF gene responsible for faciogenital dysplasia, is critical for excretory cell morphogenesis. Hum Mol Genet. 2001;10(26):3049-3062.

43. Suzuki N, et al. A putative GDP-GTP exchange factor is required for development of the excretory cell in Caenorhabditis elegans. EMBO Rep. 2001; 2(6):530-535.

44. Kant S, et al. TNF-stimulated MAP kinase activation mediated by a Rho family GTPase signaling pathway. Genes Dev. In press.

45. Wu J, Glimcher LH, Aliprantis AO. HCO3-/Cl- anion exchanger SLC4A2 is required for proper osteoclast differentiation and function. Proc Natl Acad Sci US A. 2008;105(44):16934-16939. 